\title{
The growth temperature effect on vertically aligned carbon nanotubes parameters
}

\author{
O. I. Il'in ${ }^{1}$, M. V. Il'ina ${ }^{1}$, N. N. Rudyk ${ }^{2}$, A. A. Fedotov ${ }^{2}$, O. A. Ageev ${ }^{1}$ \\ ${ }^{1}$ Southern Federal University, Research and educational center of "Nanotechnology", Russia \\ ${ }^{2}$ Southern Federal University, Laboratory of nanobiotechnologies and new materials, Russia \\ oiilin@sfedu.ru, ageev@sfedu.ru
}

PACS 61.46.Fg, 61.46.Np

DOI 10.17586/2220-8054-2018-9-1-92-94

We studied the influence of the synthesis temperature on geometric parameters and structural perfection for vertically aligned carbon nanotubes (VACNT). We established that a synthetic temperature of $750{ }^{\circ} \mathrm{C}$ allows one to obtain the lowest concentration of defects in VACNT, with a diameter of $44 \pm 3 \mathrm{~nm}$ and a height of $80 \pm 9 \mathrm{~nm}$. When temperature is increased up to $800{ }^{\circ} \mathrm{C}$, an increase of the VACNT geometric dimensions was observed, which may be due to an increase in the catalytic centers (CCs) migration rate and their integration into larger centers. Also, at $800{ }^{\circ} \mathrm{C}$, the concentration of defects in the nanotubes was increased due to the violation of carbon bonds during the acceleration of the acetylene desorption process from the surface of the sample.

Keywords: carbon nanotube, chemical vapor deposition, raman spectroscopy, catalytic centers, synthesis.

Received: 17 June 2017

\section{Introduction}

Due to unique properties of vertically aligned carbon nanotubes, they are widely applied as elements of microand nanoelectronic devices [1], fillers of various composite materials and high-strength adhesives ("dry" glue) [2]. Of particular interest are arrays of VACNT with certain geometric dimensions and conductivity. In this regard, there is a need for the synthesis of VACNT with controlled geometrical parameters, properties and structure.

The method of plasma enhanced chemical vapor deposition (PECVD), which allows to grow aligned CNTs on substrates with specially prepared catalytic centers (CC), is the most promising [3]. A large number of interrelated parameters of the PECVD method (temperature, pressure, process gas flows, etc.) have significant influence on the growth processes and properties of the VACNT [3-5]. One of the most important parameters that determines the geometric dimensions and structure of the VACNT is the synthesis temperature.

The aim of the work is to study the influence of the growth temperature of the VACNT by the PECVD method on their geometric parameters and structural perfection.

\section{Experiments and methods}

The synthesis of the VACNT was carried out by the "top-mechanism" in the PECVD module of nanotechnology complex NANOFAB NTK-9 (NT-MDT, Russia). A Si (100) wafer was used as a substrate, on which films of a buffer sublayer of $\mathrm{Cr}(20 \mathrm{~nm})$ and a catalytic layer of $\mathrm{Ni}(10 \mathrm{~nm})$ were formed by magnetron sputtering. $\mathrm{Ni} / \mathrm{Cr}$ films were used as a combination of metals in the catalyst/sublayer, because they provide high homogeneity of the parameters of carbon nanotubes [6]. The growth of VACNT was carried out in a mixture of gases $\mathrm{NH}_{3}\left(210 \mathrm{sccm}^{2}\right.$ and $\mathrm{C}_{2} \mathrm{H}_{2}(70 \mathrm{sccm})$, at a chamber pressure of 4.5 Torr for $20 \mathrm{~min}$. The synthesis temperature varied from 650 to $800{ }^{\circ} \mathrm{C}$.

We studied the VACNT samples using a scanning electron microscope (SEM) Nova NanoLab 600 (FEI, Netherlands). The diameter and height of the VACNT array were evaluated by means of statistical processing of SEM images. The structural analysis of the VACNT arrays was conducted by a Raman spectrometer Renishaw InVia Reflex (Renishaw plc, UK).

\section{Results and discussion}

The analysis of the obtained SEM images of the experimental samples showed that the VACNTs were grown by the "top-mechanism". For samples obtained at $650{ }^{\circ} \mathrm{C}$ (Fig. 1a), the height and diameter of the CNTs in the array were $65 \pm 5 \mathrm{~nm}$ and $25 \pm 3 \mathrm{~nm}$, respectively. Also presented are disoriented CNTs (Fig. 1a). Increasing the temperature to $700{ }^{\circ} \mathrm{C}$ (Fig. 1b) made possible the removal of the disoriented CNTs. In this case, the geometrical dimensions of CNTs are practically unchanged (diameter of $25 \pm 4 \mathrm{~nm}$, height of $66 \pm 5 \mathrm{~nm}$ ). This effect can be associated with a better desorption of hydrogen during the decomposition of acetylene on CC, which leads to the formation of a less "defective" layer of carbon on the $\mathrm{CC}$ surface, which does not branch and violate the vertical 
orientation of the CNT. As the temperature was increased to $750{ }^{\circ} \mathrm{C}$ (Fig. 1c), an increase in the diameter and height of the VACNT ( $44 \pm 3 \mathrm{~nm}$ and $80 \pm 9 \mathrm{~nm}$, respectively) occurred, as well as the growth of individual CNTs with a diameter of $70 \pm 3 \mathrm{~nm}$ and a height of $350 \pm 10 \mathrm{~nm}$. This may be due to the activation of diffusion exchange of atoms between the film and the substrate and, the association of small CCs into larger ones. At $800{ }^{\circ} \mathrm{C} \mathrm{(Fig.} 1 \mathrm{~d}$ ), the diameter and height of CNT arrays were $51 \pm 6 \mathrm{~nm}$ and $100 \pm 12 \mathrm{~nm}$, respectively. The growth of individual CNTs with a height of up to $600 \pm 14 \mathrm{~nm}$ and a diameter of $52 \pm 2 \mathrm{~nm}$ is also observed. The increase in the diameter of the CNT and the almost complete absence of nanotubes of diameter less than $25 \mathrm{~nm}$ indicated an ongoing process of diameter increasing, possibly resulting from $\mathrm{CC}$ or small center sublimation at this temperature.
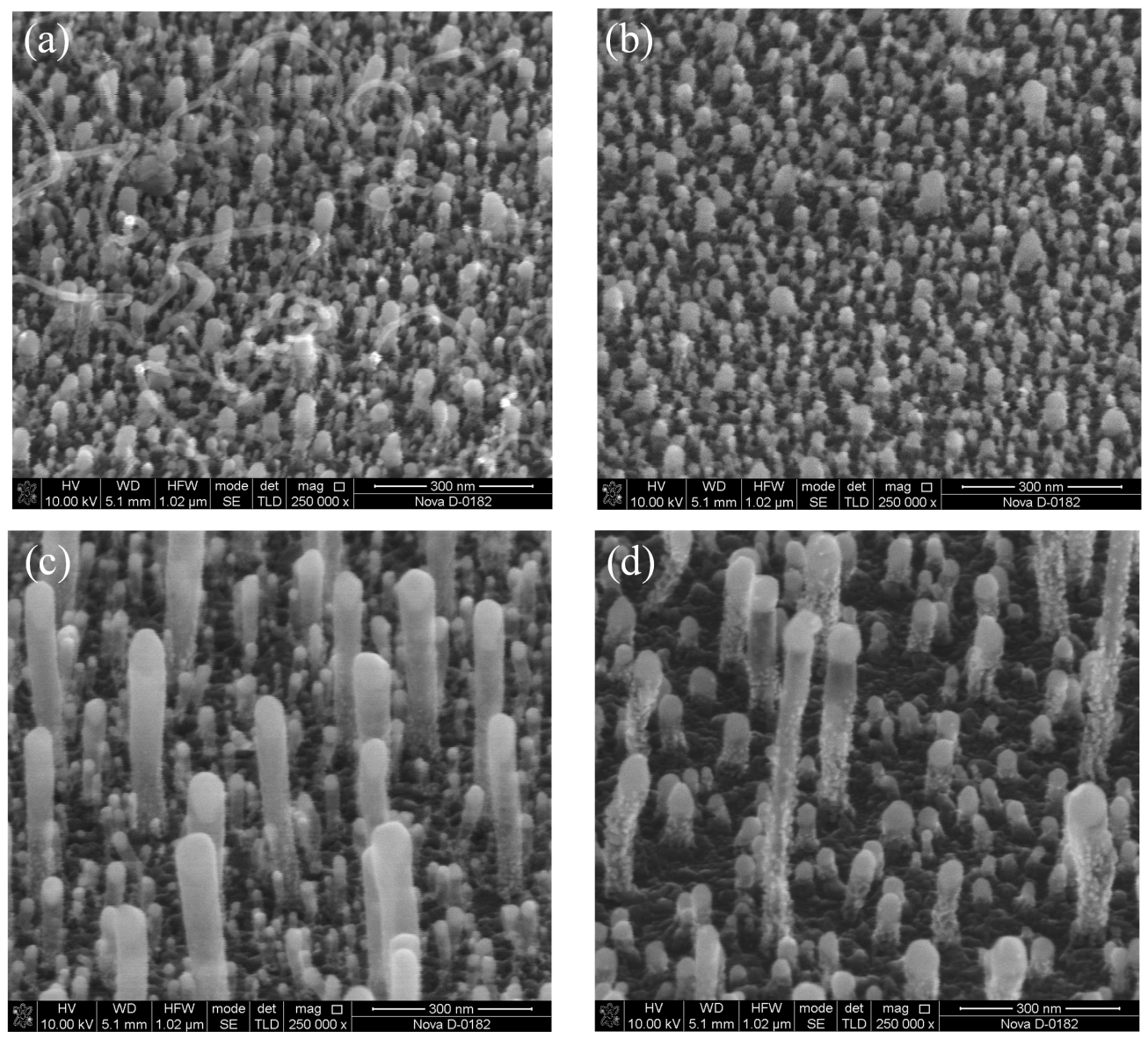

FIG. 1. SEM images of arrays of vertically aligned CNTs: $650{ }^{\circ} \mathrm{C}$ (a); $700{ }^{\circ} \mathrm{C}$ (b); $750{ }^{\circ} \mathrm{C}$ (c); $800{ }^{\circ} \mathrm{C}(\mathrm{d})$

Structural analysis of the experimental samples grown at temperatures of $650,700,750$ and $800{ }^{\circ} \mathrm{C}$ (Fig. 2) showed the presence of D- and G-mode typical for carbon nanotubes [7]. The absence of the RBM mode in the 0-200 $\mathrm{cm}^{-1}$ range in all samples indicated a multiwall type of the grown nanotubes [8].

In addition, the amplitude ratio of the $I_{D} / I_{G}$ peaks of the experimental samples allowed us to estimate the influence of the growth temperature on the defectiveness of the VACNTs and was $0.92,0.88$ and 0.90 for 700,750 and $800{ }^{\circ} \mathrm{C}$, respectively. The maximum defect concentration $\left(I_{D} / I_{G}=0.92\right)$ was observed for VACNT grown at $700{ }^{\circ} \mathrm{C}$, which is probably due to the influence of the material of the catalytic center occupying most of the volume of the entire nanotube, leading to a violation of the symmetry of the graphite layer with $\mathrm{sp}^{2}$-hybridization. Increasing the synthesis temperature to $750{ }^{\circ} \mathrm{C}$ resulted in an increase in the aspect ratio and volume of the nanotubes, reducing its defectiveness to $I_{D} / I_{G}=0.88$. With a subsequent increase in the synthesis time, the $I_{D} / I_{G}$ value increased again, which is due to the violation of carbon bonds due to the acceleration of the acetylene desorption process from the sample surface. As a consequence, the carbon-containing gas did not have time to react with the $\mathrm{CC}$ and was pumped out by the vacuum system. As a result, graphenated multi-wall carbon nanotubes were formed (Fig. 1d). 


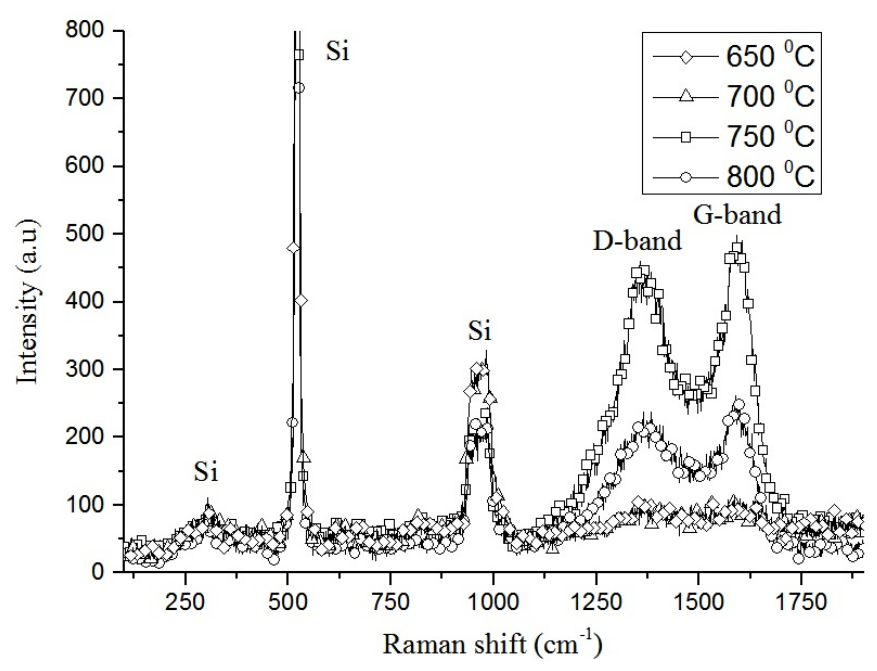

FIG. 2. Raman spectra of images with CNTs are grown at different temperatures

\section{Conclusion}

Experimental studies of the influence of the synthesis temperature of the VACNT by PECVD on their geometric parameters and structural perfection were performed. It was found that the growth temperature $750{ }^{\circ} \mathrm{C}$ allows one to obtain a CNT with the diameter of $44 \pm 3 \mathrm{~nm}$, the height of $80 \pm 9 \mathrm{~nm}$ and a minimal concentration of defects.

When temperature is increased to $800{ }^{\circ} \mathrm{C}$, increases in the diameter and height of the VACNT were observed up to $51 \pm 6 \mathrm{~nm}$ and $100 \pm 12 \mathrm{~nm}$, respectively, which may be associated with an increase in the migration rate of catalytic centers and their integration into larger centers. Structural studies have also shown smallest defects for VACNT were for those synthesized at $750{ }^{\circ} \mathrm{C}$. At $800{ }^{\circ} \mathrm{C}$, the defectiveness of nanotubes was increased due to the violation of carbon bonds during the acceleration of the acetylene desorption process from the surface of the sample. Thus, it is shown that control of the growth temperature allows optimization of the technological process of growing CNTs, to obtain nanotubes of the lowest numbers of defects and high concentration.

The obtained results can be used for the development of technological processes for the formation of vertically aligned carbon nanotubes for functional elements for emission nanoelectronics devices, memory cells and adhesion coatings.

\section{Acknowledgements}

This work was funded by the Russian Foundation for Basic Research according to the research project No. 16-29-14023 ofi_m and internal grant Internal grant of the Southern Federal University No. VnGr-07/2017-26.

\section{References}

[1] Ageev O.A., Blinov Yu.F., Il'in O.I., Konoplev B.G., Rubashkina M.V., Smirnov V.A., Fedotov A.A. Study of the resistive switching of vertically aligned carbon nanotubes by scanning tunneling microscopy. Phys. Solid State, 2015, 57, P. 825-831.

[2] Jeong H.E., Suh K.Y. Nanohairs and nanotubes: Efficient structural elements for gecko-inspired artificial dry adhesives. Nano Today, 2009, 4, P. 335-346.

[3] Saeidi M. Influence of partial pressure on base-growth of single carbon nanotube. J. Cryst. Growth, 2014, 404, P. 34-38.

[4] Shah K.A., Tali B.A. Synthesis of carbon nanotubes by catalytic chemical vapour deposition: A review on carbon sources, catalysts and substrates. Mater. Sci. Semicond. Process, 2016, 41, P. 67-82.

[5] Ageev O.A., Il'in O.I., Rubashkina M.V., Smirnov V. A., Fedotov A.A., Tsukanova O.G. Determination of the electrical resistivity of vertically aligned carbon nanotubes by scanning probe microscopy. Techn. Phys., 2015, 60(7), P. 1044-1050.

[6] Ando Y., Zhao X., Sugai T., Kumar M. Growing carbon nanotubes. Mater. Today, 2004, 7, P. $22-29$.

[7] Maultzsch J., Reich S., Thomsen C. Raman scattering in carbon nanotubes revisited. Phys. Rev. B, 2002, 65, P. 233402.

[8] Saito R., Hofmann M., Dresselhaus G., Jorio A., Dresselhaus M.S. Raman spectroscopy of graphene and carbon nanotubes. Adv. Phys., 2011, 60(3), P. 413-550. 